\title{
Arterial Stiffness Is Associated with Peripheral Sensory Neuropathy in Diabetes Patients in Ghana
}

\author{
Kwame Yeboah $\mathbb{D}^{1}{ }^{1}$ Jennifer A. Agyekum, ${ }^{2}$ Richard N. A. Owusu Mensah, ${ }^{1}$ \\ Patrick K. Affrim, ${ }^{3}$ Linda Adu-Gyamfi, ${ }^{4}$ Rita O. Doughan, ${ }^{1}$ and Afua B. Adjei ${ }^{3}$ \\ ${ }^{1}$ Department of Physiology, School of Biomedical \& Allied Health Sciences, University of Ghana, Accra, Ghana \\ ${ }^{2}$ Department of Medical Laboratory Sciences, School of Biomedical \& Allied Health Sciences, University of Ghana, Accra, Ghana \\ ${ }^{3}$ Department of Chemical Pathology, School of Biomedical \& Allied Health Sciences, University of Ghana, Accra, Ghana \\ ${ }^{4}$ Department of Medical Biochemistry, School of Biomedical \& Allied Health Sciences, University of Ghana, Accra, Ghana
}

Correspondence should be addressed to Kwame Yeboah; melvinky@gmail.com

Received 24 July 2017; Revised 30 November 2017; Accepted 12 December 2017; Published 31 January 2018

Academic Editor: Laurent Crenier

Copyright (c) 2018 Kwame Yeboah et al. This is an open access article distributed under the Creative Commons Attribution License, which permits unrestricted use, distribution, and reproduction in any medium, provided the original work is properly cited.

\begin{abstract}
Objective. Peripheral sensory neuropathy (PSN) is among microvascular complications of diabetes that make patients prone to ulceration and amputation. Arterial stiffness is a predictor of cardiovascular diseases and microvascular complications associated with diabetes. We investigated the association between PSN and arterial stiffness, measured as aortic pulse wave velocity (PWVao) and cardio-ankle vascular index (CAVI). Method. In a case-control design, arterial stiffness was measured in 240 diabetes patients and 110 nondiabetic control. Large-fibre nerve function was assessed by vibration perception threshold (VPT) using a neurothesiometer. PSN was defined as the VPT > 97.5th percentile from age- and gender-adjusted models in nondiabetic controls. Results. The overall prevalence of PSN was $16.6 \%$ in the entire study participants. Compared to non-PSN participants, PSN patients had higher levels of PWVao $(9.5 \pm 1.7$ versus $8.7 \pm 1.2 \mathrm{~m} / \mathrm{s}, p=0.016)$ and CAVI $(8.4 \pm 1.3$ versus $7.6 \pm 1.1, p=$ 0.001). In multiple regression models, VPT was associated with PWVao $(\beta=0.14, p=0.025)$ and CAVI $(\beta=0.12, p=0.04)$. PSN patients had increased odds of CAVI (OR $=1.51(1.02-2.4), p=0.043)$, but not PWVao (OR=1.25 $(0.91-1.71), p=0.173)$. Conclusion. PWVao and CAVI were associated with VPT and PSN in diabetes patients in Ghana. Patients having PSN have increased odds of CAVI, independent of other conventional risk factors.
\end{abstract}

\section{Introduction}

Diabetes is now common in sub-Saharan African population and associated with macrovascular and microvascular complications [1,2]. Cardiovascular diseases are among the major causes of death in diabetes patients; however, studies have shown that microvascular disease is also associated with excess mortality [3]. Coexistence of cardiovascular and microvascular diseases in diabetes patients is associated with higher mortality $[4,5]$. Peripheral sensory neuropathy (PSN) in the lower limbs is a classic example of microvascular complication of diabetes, with a high risk for nontraumatic limb amputation, which occurs in $1-2 \%$ of diabetic patients and necessitates extreme cost [6].
Diabetes patients in Ghana have abnormalities in arterial function that can be measured using aortic pulse wave velocity (PWVao) or cardio-ankle vascular index (CAVI) [7, 8]. Both PWVao and CAVI measure arterial stiffness, with CAVI assessing the stiffness in long range of arterial segment (from the aortic root to the ankle arteries), as opposed to PWVao, which assesses the stiffness in the descending aorta, from the aortic root to the bifurcation. Arterial stiffness predicts cardiovascular and all-cause mortality in diabetes patients $[9,10]$. The association between arterial stiffness and PSN in diabetes patients in sub-Saharan Africa is unknown. We, therefore, investigated the levels of various indices of arterial stiffness in PSN patients and the association between PSN and arterial stiffness. We hypothesize that, compared to non-PSN individuals, PSN patients have higher levels of arterial stiffness. 


\section{Materials and Methods}

2.1. Study Design and Subjects. This study was a case control design, conducted within the period of December 2012 to June 2013, at the Korle-Bu Teaching Hospital in Accra, which is a 1500-bed tertiary hospital and serves as the main referral hospital in Ghana. The study population were selected from two sources: (1) diabetes patients, selected systematically as every 3rd consecutive patient visiting the diabetes clinic and consented to take part in the study, and (2) nondiabetic individuals, invited from the surrounding communities and conveniently recruited into the study. Vibration perception threshold and arterial stiffness were assessed in all the study participants. Individuals with nontraumatic limb amputation and those unable to comprehend and comply with the protocol requirements (psychological and/or cognitive disorders, failure to cooperate, and failure to sign the informed consent document) were excluded from the study. In all, 350 subjects, comprising 240 diabetes patients and 110 nondiabetic individuals, were screened for PSN. The study was approved by the University of Ghana Medical School Ethical and Protocol Review Committee (protocol ID number: MS-Et/M.2 - P.4.10/2012-2013) and all participants gave written informed consent after the procedures involved in the study were thoroughly explained to them, following the general recommendations of the Declaration of Helsinki.

2.1.1. Anthropometric Measurements. Using standard protocols [11], body weight was determined twice using a homologated electronic scale (Seca 770, Hamburg, Germany) following due calibration (precision $\pm 0.1 \mathrm{~kg}$ ), with the patient wearing light clothing and shoes removed. Height was also measured with a portable system (Seca 222, Hamburg, Germany) with the patient shoeless in the upright position. Body mass index (BMI) was calculated as weight $(\mathrm{kg})$ divided by height squared $\left(\mathrm{m}^{2}\right)$. Waist circumference was measured with a nonelastic tape measure at the upper border of the iliac crest, parallel to the floor without compressing the skin. Body composition monitor (BF508, Omron Healthcare Inc., Vernon Hills, IL, USA) was used to assess body fat percentage.

2.1.2. Biochemical Analysis. Blood samples were drawn in the morning, after 8-12 hours of overnight fasting, into plain vacuum tubes to measure plasma lipids and fluoride oxalate tubes for glucose levels. FPG, 2h-PPG, total cholesterol, high-density lipoprotein cholesterol (HDL), and triglyceride levels were analysed by colorimetric enzymatic assays using BS 120 chemical autoanalyser (Mindray, China) and commercial reagents (Randox Laboratory Reagents, UK). Lowdensity lipoprotein cholesterol levels were calculated using Friedewald's formula [12]. All analyses were performed at the Diabetes Research and Chronic Disease Reference Laboratory.

2.2. Arterial Stiffness Assessment. Arteriograph (TensioMed Kft., Hungary) was used to measure aortic pressure indices, aortic PWV (PWVao), and aortic augmentation index (AIx), with the subject lying supine for at least 10 minutes in a temperature-controlled room $\left(22 \pm 2^{\circ} \mathrm{C}\right)$. The Arteriograph cuff was applied on the right arm over the brachial artery to detect arterial wall oscillations in the upper arm using the "stop-flow" principle previously described [13]. To determine PWVao, the Arteriograph uses the physiological behaviour of the wave reflection; the ejected direct (first systolic) pulse wave is reflected back mostly from the aortic bifurcation. The device measures the time interval between the peaks of the direct (first) and reflected (late) systolic wave (return time). For both the invasive and noninvasive PWVao calculation, the distance from the sternal notch (jugulum) to the upper edge of the pubic bone (symphysis) is used because this provides the nearest value of the true aortic path length $[14,15]$. To avoid overestimation of aortic path length by body surface contours, a specialised body calliper was used to measure the jugulum-symphysis distance.

The aortic PWV was calculated by using the following formula:

$$
\text { Aortic PWV }(\mathrm{m} / \mathrm{s})=\frac{2(\text { jugulum }- \text { symphysis distance })}{\text { return time }} .
$$

Augmentation index (AIx) is computed using the algorithm below:

$$
\mathrm{AIx}=\frac{P_{2}-P_{1}}{\mathrm{PP}} \times 100
$$

where $P_{1}$ is the amplitude of the first (direct) wave, $P_{2}$ is the amplitude of the late (reflected) systolic wave, and PP is the pulse pressure.

CAVI and heart-ankle (ha) PWV were measured using the Vasera $1500 \mathrm{~N}$ (Fukuda-Denshi, Japan) with the participant resting supine for at least 10 minutes before the measurement. Electrocardiogram electrodes were placed on both wrists, a microphone for detecting heart sounds placed on the sternum, and cuffs were wrapped around the upper arms and the ankles. CAVI values were computed automatically. Briefly, CAVI corresponds to the stiffness parameter $\beta$, calculated from values of ha-PWV and BP as follows:

$$
\beta=\left(\frac{2 \rho}{\Delta P}\right) \cdot\left[\ln \left(\frac{P_{\mathrm{s}}}{P_{\mathrm{d}}}\right)\right] \mathrm{PWV}^{2},
$$

where $\rho$ indicates blood density; $\Delta P$, pulse pressure; ln, natural log; $P_{\mathrm{s}}$, systolic BP; and $P_{\mathrm{d}}$, diastolic BP $[16,17]$. CAVI and Arteriograph measurements were performed in a random order using a digital algorithm.

2.3. Neurothesiometry. Neurothesiometry was performed using hand-held neurothesiometer (Horwell Neurothesiometer, Scientific Laboratory Supplies Ltd., Nottingham, $\mathrm{UK})$ to the read vibration perception threshold (VPT) from the apex of the big toe of both legs, with the subject in a supine position, feet elevated with pillow support, and eyes closed. The neurothesiometer is a validated batteryoperated diagnostic instrument that assesses sensitivity thresholds to vibration corresponding to a particular voltage at various sites on the body surface. On the basis of 
TABLE 1: General characteristics of study participants by diabetes status.

\begin{tabular}{|c|c|c|c|c|}
\hline & All participants $(n=350)$ & T2DM $(n=240)$ & Non-DM $(n=110)$ & $p$ \\
\hline Females, $n(\%)$ & $175(50)$ & $108(45)$ & $67(60.1)$ & 0.15 \\
\hline Hypertension, $n(\%)$ & $179(52.6)$ & $137(57.1)$ & $42(38.2)$ & \\
\hline Age (yrs) & $54.1 \pm 10.2$ & $53.7 \pm 10.1$ & $54.6 \pm 10.3$ & 0.54 \\
\hline Duration of diabetes (yrs) & $10.4 \pm 7.8$ & $10.4 \pm 7.8$ & & \\
\hline Weight (kg) & $79.5 \pm 14.9$ & $79.9 \pm 15.5$ & $79 \pm 14.3$ & 0.672 \\
\hline Height (cm) & $166 \pm 8.4$ & $167 \pm 8$ & $164 \pm 9$ & 0.061 \\
\hline BMI $\left(\mathrm{kg} / \mathrm{m}^{2}\right)$ & $29.1 \pm 5.7$ & $28.9 \pm 5.9$ & $29.4 \pm 5.5$ & 0.571 \\
\hline Waist circumference $(\mathrm{cm})$ & $98 \pm 14$ & $99 \pm 12$ & $96 \pm 15$ & 0.073 \\
\hline Waist-hip ratio & $0.91 \pm 0.11$ & $0.92 \pm 0.07$ & $0.9 \pm 0.14$ & 0.382 \\
\hline Systolic BP (mmHg) & $139 \pm 30$ & $141 \pm 26$ & $135 \pm 34$ & 0.174 \\
\hline Diastolic BP (mmHg) & $83 \pm 13$ & $83 \pm 13$ & $82 \pm 14$ & 0.594 \\
\hline Pulse BP (mmHg) & $59 \pm 14$ & $59 \pm 14$ & $58 \pm 13$ & 0.485 \\
\hline Heart rate $(\mathrm{bpm})$ & $71 \pm 17$ & $75 \pm 13$ & $65 \pm 19$ & $<0.01$ \\
\hline $\mathrm{FPG}(\mathrm{mmol} / \mathrm{l})$ & $6.9 \pm 3.2$ & $8.4 \pm 2.9$ & $5 \pm 2.5$ & $<0.01$ \\
\hline 2h-PPG (mmol/l) & $7.8 \pm 1.4$ & & $7.8 \pm 1.4$ & \\
\hline Triglycerides (mmol/l) & $1.1 \pm 0.5$ & $1.1 \pm 0.5$ & $1.2 \pm 0.6$ & 0.586 \\
\hline Total cholesterol (mmol/l) & $4.7 \pm 1.5$ & $5.5 \pm 1.4$ & $3.9 \pm 1.1$ & $<0.001$ \\
\hline HDL cholesterol (mmol/l) & $0.9 \pm 0.2$ & $0.7 \pm 0.2$ & $1.2 \pm 0.4$ & 0.025 \\
\hline LDL cholesterol (mmol/l) & $3.2 \pm 1.4$ & $3.9 \pm 1.3$ & $2.7 \pm 1.4$ & $<0.001$ \\
\hline PWVao (m/s) & $8.8 \pm 1.6$ & $9 \pm 1.5$ & $7.8 \pm 1.7$ & 0.006 \\
\hline AIx (\%) & $29 \pm 14.6$ & $27.1 \pm 14.7$ & $34 \pm 12.9$ & $<0.001$ \\
\hline Aortic systolic BP (mmHg) & $143 \pm 29$ & $137 \pm 28$ & $145 \pm 29$ & 0.034 \\
\hline Aortic pulse BP (mmHg) & $55 \pm 24$ & $58 \pm 19$ & $63 \pm 26$ & 0.082 \\
\hline CAVI & $7.6 \pm 1.3$ & $7.8 \pm 1.1$ & $7.1 \pm 1.1$ & 0.004 \\
\hline haPWV $(\mathrm{m} / \mathrm{s})$ & $7.7 \pm 1.7$ & $7.9 \pm 1.9$ & $7.5 \pm 1.2$ & 0.094 \\
\hline VPT (V) & $11.9 \pm 6.2$ & $15.1 \pm 7.8$ & $7.3 \pm 3.8$ & $<0.001$ \\
\hline PSN, $n(\%)$ & $58(16.6)$ & $47(19.4)$ & $11(10)$ & $<0.001$ \\
\hline
\end{tabular}

the method of limits, participants were asked to indicate when they first perceived vibration sensation after the stimulus was applied to the distal pulp of the toe. The intensity of the stimulus was gradually increased at a rate of $0.5 \mathrm{~V} / \mathrm{s}$ from null to a voltage at which vibration was first detected. VPT was performed on each participant about 3-5 times and, at least, three VPTs that differed $\leq 5 \mathrm{~V}$ were averaged and used for analysis. A null stimulus test was randomly performed to ensure that the participant understands and adheres to the test procedure. Participants who failed to provide 3 consistent values of VPT within $5 \mathrm{~V}$ after seven measurements were excluded from the analysis, considered as having conflicting VPTs.

2.4. Statistical Analysis. Continuous data were analysed with the Shapiro-Wilk test to determine their distribution, and skewed variables were logarithmically transformed before analysis. The cut-offs for PNS were computed as the $\mathrm{VPT} \geq 97.5$ th percentile from age- and gender-adjusted regression models in the nondiabetic controls. From the regression models, $\mathrm{VPT}>24 \mathrm{~V}$ in males and $>21 \mathrm{~V}$ in females were used to define PNS. Variables with normal distribution were presented as mean \pm standard deviation and analysed using Student's $t$-test or analysis of variance, as deemed appropriate. Variables with nonnormal distribution were presented as median and interquartile range and analysed using Mann-Whitney $U$ test or Kruskal-Wallis test, as deemed appropriate. Categorical data were analysed by the $\chi^{2}$ test. Zero-order and partial Pearson's correlation tests were used to test the association between VPT versus arterial stiffness indices and patient characteristics. Multivariable linear and logistic regression models were performed to analyse the association between VPT/PSN and indices of arterial stiffness.

\section{Results}

The prevalence of PSN in our study population was $16.6 \%$, higher in diabetes patients. Compared to nondiabetic individuals, diabetes patients have higher heart rate, pulse pressure, total and LDL cholesterol, PWVao, aortic systolic BP, aortic pulse BP, CAVI, ha-PWV, and VPT. Diabetes patients had lower HDL cholesterol and AIx (Table 1). Compared to non-PSN participants, PSN patients were taller and had higher duration of diabetes, higher systolic and pulse BP, and higher heart rate (Table 2). VPT correlated positively 
TABle 2: Comparison of clinical features of study participants by PSN status.

\begin{tabular}{|c|c|c|c|}
\hline & $\begin{array}{c}\text { No PSN } \\
(n=292)\end{array}$ & $\begin{array}{c}\text { PSN } \\
(n=58)\end{array}$ & $p$ \\
\hline Age (yrs) & $\begin{array}{c}54.3 \\
\pm 10.2\end{array}$ & $\begin{array}{c}52.6 \\
\pm 10.4\end{array}$ & 0.529 \\
\hline Females, $n(\%)$ & $154(52.7)$ & $21(36.2)$ & 0.063 \\
\hline Diabetes, $n(\%)$ & $193(66.1)$ & $47(81)$ & 0.011 \\
\hline Hypertension, $n(\%)$ & $117(50)$ & $35(60.3)$ & 0.032 \\
\hline Duration of diabetes & $7.7 \pm 6.6$ & $14.8 \pm 6.8$ & 0.001 \\
\hline Previous smoking, $n$ (\%) & $38(13)$ & $13(22.4)$ & 0.15 \\
\hline Weight (kg) & $79 \pm 14.9$ & $84 \pm 15$ & 0.215 \\
\hline Height $(\mathrm{cm})$ & $165 \pm 8$ & $175 \pm 7$ & $<0.001$ \\
\hline BMI $\left(\mathrm{kg} / \mathrm{m}^{2}\right)$ & $29.2 \pm 5.7$ & $27.6 \pm 5.2$ & 0.257 \\
\hline Body fat (\%) & $\begin{array}{c}35.1 \\
\pm 12.6\end{array}$ & $28 \pm 11.5$ & 0.037 \\
\hline Visceral fat (\%) & $11.2 \pm 4.1$ & $12.4 \pm 6$ & 0.324 \\
\hline Waist circumference $(\mathrm{cm})$ & $98 \pm 13$ & $98 \pm 12$ & 0.892 \\
\hline Waist-hip ratio & $0.91 \pm 0.1$ & $\begin{array}{c}0.92 \\
\pm 0.05\end{array}$ & 0.825 \\
\hline Systolic BP (mmHg) & $138 \pm 30$ & $154 \pm 18$ & 0.006 \\
\hline Diastolic BP (mmHg) & $82 \pm 13$ & $87 \pm 15$ & 0.176 \\
\hline Pulse BP (mmHg) & $58 \pm 14$ & $66 \pm 8$ & 0.002 \\
\hline Heart rate $(\mathrm{bpm})$ & $70 \pm 17$ & $80 \pm 7$ & $<0.001$ \\
\hline FPG (mmol/l) & $6.9 \pm 3.3$ & $6.5 \pm 1.9$ & 0.387 \\
\hline Triglycerides (mmol/l) & $1.1 \pm 0.5$ & $1 \pm 0.4$ & 0.205 \\
\hline Total cholesterol (mmol/l) & $4.7 \pm 1.4$ & $4.5 \pm 1.8$ & 0.506 \\
\hline HDL cholesterol (mmol/l) & $0.7 \pm 0.2$ & $0.7 \pm 0.3$ & 0.913 \\
\hline LDL cholesterol (mmol/l) & $3.3 \pm 1.4$ & $3.2 \pm 1.5$ & 0.825 \\
\hline
\end{tabular}

with age, duration of diabetes, waist-hip ratio, and fasting plasma glucose and negatively with body fat (Table 3 ).

With respect to the indices of arterial stiffness, compared to non-PSN participants, PSN patients had higher means of PWVao, (Figures 1(a) and 1(b)). In the entire participants, log VPT was associated with log PWVao in zero-order $(r=0.14, p=0.026)$ and age and gender partial $(r=0.12, p=0.048)$ correlations. Similarly, log VPT was associated with CAVI in zero-order $(r=0.24, p<0.001)$ and age and gender partial $(r=0.12, p=0.04)$ correlations. When the participants were grouped by diabetes status, log VPT correlated with log PWVao and CAVI in diabetes patients, but not significant in nondiabetes participants (Figures 2(a) and 2(b)).

In multivariable linear regression analysis, with confounders forced into the model, VPT was associated with PWVao, CAVI, and haPWV. Other factors like age, body height, and diabetes status were also associated with VPT (Table 4). In logistic regression models, PSN patients have increased odds of change in PWVao and CAVI in unadjusted models. In adjusted models, PSN patients have increased odds of change in CAVI and decreased odds of change in AIx. The contributions of other variables in the models were indicated in the footnote (Table 5).
TABLE 3: Correlation between VPT and clinical parameters of participants.

\begin{tabular}{lcc}
\hline & $r$ & VPT \\
\hline Age & 0.23 & $p$ \\
Duration of diabetes & 0.28 & 0.002 \\
Body mass index & -0.12 & 0.004 \\
Body fat & -0.18 & 0.110 \\
Visceral fat & 0.07 & 0.015 \\
Waist circumference & 0.07 & 0.391 \\
Waist-hip ratio & 0.17 & 0.366 \\
Waist-height ratio & -0.05 & 0.021 \\
Fasting plasma glucose & 0.17 & 0.542 \\
2h-PPG & 0.07 & 0.018 \\
Total cholesterol & 0.05 & 0.574 \\
Triglycerides & -0.01 & 0.510 \\
HDL cholesterol & 0.008 & 0.902 \\
LDL cholesterol & -0.01 & 0.293 \\
\hline
\end{tabular}

\section{Discussion}

The findings of this study showed that compared to participants without PSN, PSN patients had higher levels of PWVao and CAVI. Also, VPT increases significantly with PWVao, CAVIs, and ha-PWV in multiple regression models, after adjusting for confounders. However, only CAVI was increased in the multivariable logistic regression model. These findings are similar to other studies that reported association between neuropathy and arterial stiffness; though most of these studies utilized subjective neurological examination for diagnosis of neuropathy $[18,19]$. A recent crosssectional study reported that carotid-femoral PWV, which is the gold standard for arterial stiffness, was associated with severity of neuropathy assessed using the neuropathy symptom score instrument [20]. Yokoyama et al. [18] reported that ba-PWV, an index similar to ha-PWV, increases together with levels of neuropathy assessed by neuropathic symptoms and tuning fork vibration. In Korean diabetes patients, Kim et al. [19] reported that neuropathy, assessed as neuropathic symptoms and current perception threshold, was associated with CAVI. Likewise, the Rio de Janeiro Type 2 Diabetes Cohort Study, which utilized the longitudinal design, reported that patients with high carotid-femoral PWV $(>10 \mathrm{~m} / \mathrm{s})$ had twice the risk of being clinically diagnosed with peripheral neuropathy during a median followup of 6.2 years [21]. As indicated by adjusted $R^{2}$ values in multiple linear regression models, the indices of arterial stiffness might be able to explain up to $25 \%$ of the variations in the VPT in our study participants. Also, the association between CAVI and PSN in multivariable logistic regression implies that stiffness in the long arterial path might be a significant determinant of PSN.

The link between arterial stiffness and neuropathy can be explained by the unique anatomy of vascular supply to peripheral nerves. Peripheral nerves, in contrast to the 


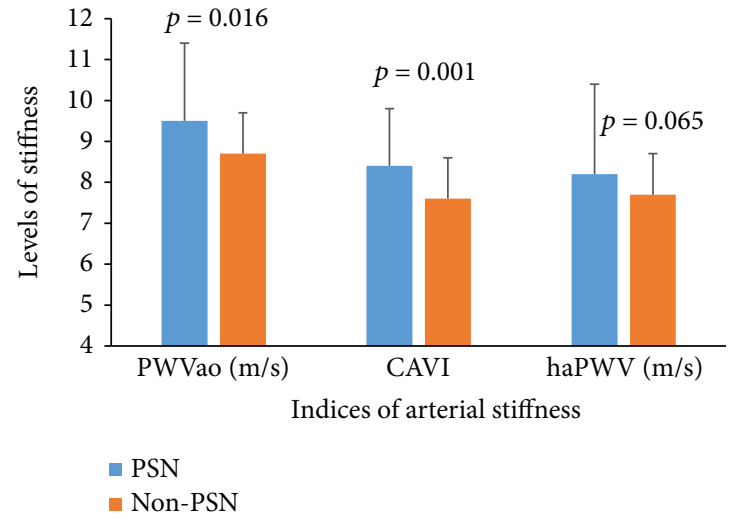

(a)

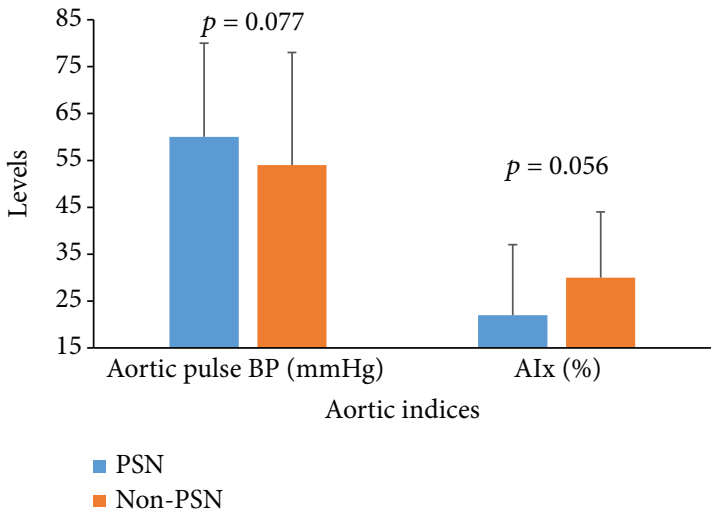

(b)

FIGURE 1: (a) Comparison of arterial stiffness indices by PSN status. Bars represent means and error bars represent standard deviations. (b) Comparison of aortic indices by PSN status. Bars represent means and error bars represent standard deviations.

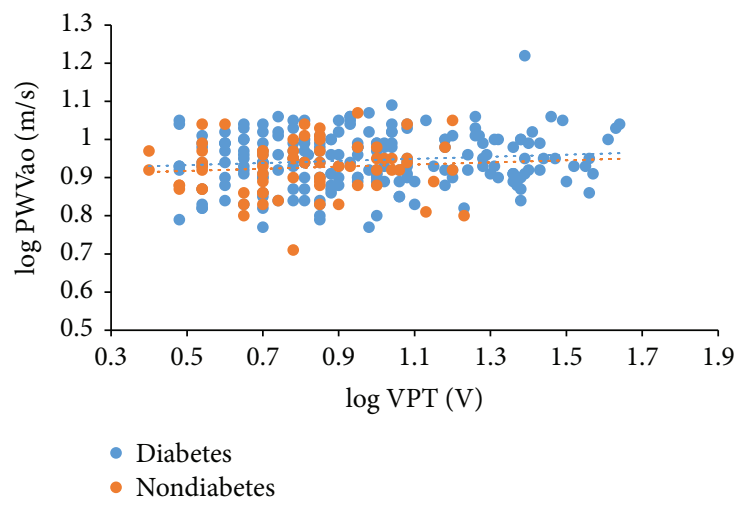

(a)

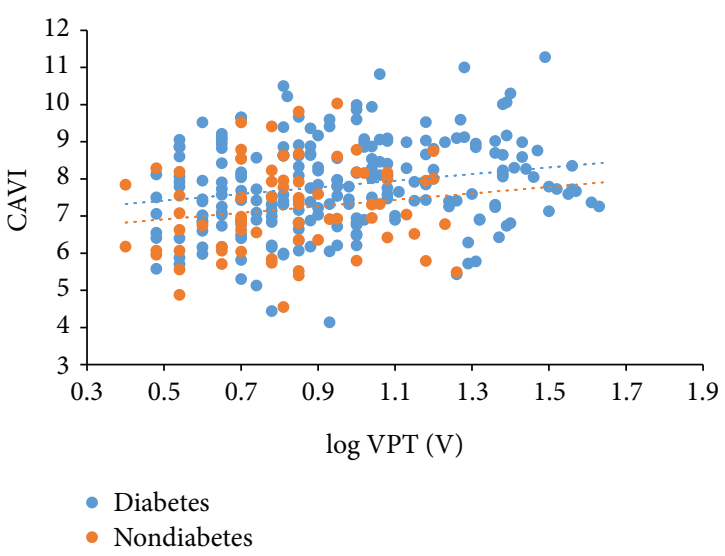

(b)

FIgure 2: (a) Correlation between log VPT and log PWVao in diabetes and nondiabetes participants. Data analysed by Pearson's correlation test. In nondiabetes participants, log VPT do not correlate with $\log$ PWVao in zero-order $(r=0.13, p=0.313)$ and age and gender partial $(r=-0.1, p=0.444)$ correlations. In diabetes patients, log VPT correlated with log PWVao in zero-order $(r=0.14, p=0.013)$ and age and gender partial $(r=0.16, p=0.004)$ correlations. (b) Correlation between log VPT and CAVI in diabetes and nondiabetes participants. Data analysed by Pearson's correlation test. In nondiabetes participants, log VPT do not correlate with CAVI in zero-order $(r=0.16, p=0.186)$ and age and gender partial $(r=-0.05, p=0.69)$ correlations. In diabetes patients, $\log$ VPT correlated with log CAVI in zero-order $(r=0.21, p=0.002)$ and nonsignificant in age and gender partial $(r=0.12, p=0.067)$ correlations.

TABLE 4: Multiple regression models of VPT and indices of arterial stiffness.

\begin{tabular}{lccc}
\hline & Unstandardized B & Standardised coefficient $(\beta)$ & Adjusted $R^{2}$ \\
\hline PWVao $^{1}$ & $0.026 \pm 0.104$ & 0.136 & $0.25 \pm 0.24$ \\
CAVI $^{2}$ & $0.023 \pm 0.011$ & 0.121 & $0.26 \pm 0.22$ \\
ha-PWV & $0.032 \pm 0.013$ & 0.141 & $0.24 \pm 0.23$ \\
AIx & $-0.004 \pm 0.001$ & -0.1 & $0.25 \pm 0.24$ \\
Aortic systolic BP & $0.009 \pm 0.001$ & 0.092 & $0.24 \pm 0.23$ \\
Aortic pulse BP & $0.002 \pm 0.001$ & 0.052 & $0.25 \pm 0.23$ \\
\hline
\end{tabular}

Adjusted for age, gender, height, BMI, waist circumference, diabetes, hypertension, alcohol, and smoking. PWVao: aortic pulse wave velocity; CAVI: cardioankle vascular index; ha-PWV: heart-ankle pulse wave velocity; AIx: aortic augmentation index; BP: blood pressure. ${ }^{1}$ Significant variables in the model were age $(\beta=0.32, p<0.001)$, body height $(\beta=0.45, p<0.001)$, and diabetes status $(\beta=0.17, p=0.003)$. ${ }^{2}$ Significant variables in the model were age $(\beta=0.34, p<0.001)$, body height $(\beta=0.46, p<0.001)$, and diabetes status $(\beta=0.17, p=0.001) .{ }^{3}$ Significant variables in the model were age $(\beta=0.31$, $p<0.001)$, body height $(\beta=0.48, p<0.001)$, and diabetes status $(\beta=0.19, p=0.001)$. 
TABLE 5: Logistic regression models of PSN and indices of arterial stiffness.

\begin{tabular}{|c|c|c|c|c|}
\hline & Unadjusted OR (95\% CI) & $p$ & Adjusted OR (95 CI) & $p$ \\
\hline $\mathrm{PWVao}^{1}$ & $1.38(1.06-1.79)$ & 0.017 & $1.25(0.91-1.71)$ & 0.173 \\
\hline $\mathrm{CAVI}^{2}$ & $1.78(1.26-2.52)$ & 0.001 & $1.51(1.02-2.4)$ & 0.043 \\
\hline ha-PWV ${ }^{3}$ & $1.26(0.93-1.76)$ & 0.137 & $1.47(0.98-2.22)$ & 0.066 \\
\hline $\mathrm{AIx}^{4}$ & $0.97(0.95-1.01)$ & 0.056 & $0.95(0.91-0.98)$ & 0.007 \\
\hline Aortic systolic $\mathrm{BP}^{5}$ & $1.01(1-1.02)$ & 0.11 & $0.97(0.93-1)$ & 0.072 \\
\hline Aortic pulse $\mathrm{BP}^{6}$ & $1.01(0.99-1.03)$ & 0.238 & $0.99(0.97-1.02)$ & 0.76 \\
\hline
\end{tabular}

Adjusted for age, gender, height, BMI, waist circumference, diabetes, hypertension, alcohol, and smoking. PWVao: aortic pulse wave velocity; CAVI: cardioankle vascular index; ha-PWV: heart-ankle pulse wave velocity; AIx: aortic augmentation index; BP: blood pressure. The other significant variables in the multivariable-adjusted models were as follows: ${ }^{1}$ Age $(\mathrm{OR}=1.08(1.02-1.15), p=0.01)$, body height $(\mathrm{OR}=1.18(1.09-2.28)$, $p<0.001)$, and diabetes status $(\mathrm{OR}=8.74(2.01-12.14), p=0.042) .{ }^{2} \mathrm{Gender}(\mathrm{OR}=3.65(1.05-12.99), p=0.046)$ and body height $(\mathrm{OR}=1.18(1.09-2.28), p<0.001) .{ }^{3} \mathrm{Age}(\mathrm{OR}=1.06$ (1.01-1.12), $p=0.037)$, male gender (OR=5.1 (1.35-14.65), $p=0.017)$, and body height $(\mathrm{OR}=1.19(1.1-2.29), p<0.001) .{ }^{4} \mathrm{Age}(\mathrm{OR}=1.12(1.05-1.19)$, $p=0.037)$, male gender $(\mathrm{OR}=3.6(1.49-13.45), p=0.012)$, and body height $(\mathrm{OR}=1.18(1.09-2.29), p<0.001) .{ }^{5} \mathrm{Age}(\mathrm{OR}=1.09(1.03-1.16), p=0.004)$, male gender $(\mathrm{OR}=3.98(1.04-12.17), p=0.044)$, and body height $(\mathrm{OR}=1.18(1.08-2.28), p<0.001) .{ }^{6} \mathrm{Age}(\mathrm{OR}=1.08(1.03-1.15), p=0.004)$, male gender $(\mathrm{OR}=4.28(1.19-13.38), p=0.026)$, body height $(\mathrm{OR}=1.18(1.09-2.27), p<0.001)$, and diabetes status $(\mathrm{OR}=4.28(1.19-9.54), p=0.046)$.

central nervous system, are supplied by the upper branches of arteries supplying musculature of the limbs. Hence, it could be expected that stiffness in the peripheral arteries can affect the peripheral nerves as well. Also, as reported by Smith et al. [22], vascular supply to peripheral nerves are sparse and lacks autoregulation, making the nerves vulnerable to ischemia as a result of stiffness in elastic arteries. In addition, Edmonds et al. [23] and Young et al. [24] observed higher levels of arterial medial calcification in diabetes patients with neuropathic symptoms when compared to diabetes patients without neuropathy. This means that diabetes causes arterial medial calcification, resulting in increased arterial stiffness, which might also lead to neuropathy [25].

The findings of this study showed that the prevalence of neuropathy was $16.4 \%$ higher in diabetes patients. VPT assess the function of large-fibre nerves, and hence, high VPT may be an indication of large-fibre nerve damage [4]. This method had been found to predict foot ulceration in high-risk subjects [26]. The prevalence of PSN in diabetes patients in Sweden was 34\% [27] and 25.5\% in Singapore [28]. The low prevalence of PNS in our study might be that diabetes-related neuropathy is presented differently in diabetes patients in Ghana. Also, there might be a possibility that the subjects did not understand the protocol well and/or gave a false feedback about perceived vibration sensation [29]. The utility and validity of neurothesiometer to detect PSN in subSaharan Africans need to be determined in a longitudinal study design. The major limitation of neurothesiometry in screening for PSN is the subject's dependency, and this can be overcome by using the objective form of assessment such as the nerve conduction study [30].

The findings of this study also show that VPT correlated with age, duration of diabetes, body fat, WHR, and FPG. Also, in the multivariable analyses, age, gender, and body height emerged as significant predictors of PSN and VPT. This is similar to the findings of Bergenheim et al. [31], who reported an association of diabetic neuropathy with age, body height, and duration of diabetes. Tesfaye et al. [32] in the EURO-Diab group reported that blood glucose control, duration of diabetes, and hyperlipidaemia were significant risk factors for the development of neuropathy in type 1 diabetes patients. Contrary to the findings of Tesfaye et al., plasma lipids did not correlate with VPT in this study. Our findings were similar to that of Yagihashi et al. [33], who reported that plasma levels of triglycerides or cholesterol were not associated with diabetic neuropathy.

4.1. Limitations of the Study. In this study, PSN was assessed employing quantitative vibration testing in the form of VPT. VPT assess the acuity of somatosensory pathways responsible for transmitting information induced by cutaneous vibratory stimuli, being neuroselective for large and myelinated $\mathrm{A} \alpha$ and $\mathrm{A} \beta$ sensory fibres [34]. Therefore, VPT fails to assess the functional status of small and medium nerves. Another notable caution about VPT is that it is used to assess probable neuropathy and nerve conduction studies are required for confirmation [35]. This study was a cross-sectional design, and hence, we cannot infer causation from our findings, whether arterial stiffness precedes the development of neuropathy or neuropathy causes arterial dysfunction in diabetes patients. Also, the participants of the study were recruited from a tertiary health facility, implying that the findings may differ from diabetes patients from primary healthcare facilities. Therefore, we cannot infer the conclusions to the entire Ghanaian population. It will be interesting for future studies to investigate the association between arterial stiffness and PSN in diabetes patients from other levels of health care facilities, and utilizing the longitudinal study design.

\section{Conclusion}

Our findings indicate that arterial stiffness, measured as PWVao and CAVI, was associated with VPT and PSN in diabetes patients in Ghana. Patients having PSN have increased odds of CAVI, independent of other conventional risk factors. 


\begin{abstract}
Abbreviations
PSN: $\quad$ Peripheral sensory neuropathy

VPT: Vibration perception threshold

PWVao: Aortic pulse wave velocity

CAVI: Cardio-ankle vascular index

ha-PWV: Heart-ankle pulse wave velocity

AIx: Augmentation index

BP: $\quad$ Blood pressure.
\end{abstract}

\section{Data Access}

The dataset supporting the conclusion of this study is available for systematic review and meta-analysis upon request.

\section{Ethical Approval}

The study was conducted in conformity with the Helsinki Declaration on Human Experimentation, 1964, with subsequent revisions, latest Seoul, October 2008. Ethical approval for the study was granted by the Ethics and Protocol Review Committee of the College of Health Science, University of Ghana (protocol ID number: MSEt/M.2 - P.4.10/2012-2013).

\section{Consent}

All the participants provided voluntary written consent before being recruited into the study.

\section{Conflicts of Interest}

The authors declare that they have no competing/financial interests.

\section{Authors' Contributions}

Kwame Yeboah contributed to the conception, design, data collection and analysis and drafting of the manuscript and bears the primary responsibility for the content of the manuscript. Richard N. A. Owusu Mensah, Jennifer A. Agyekum, Afua B. Adjei, Patrick K. Affrim, and Linda Adu-Gyamfi were involved in the revision of the manuscript. All the authors read and approved the content of the manuscript.

\section{References}

[1] K. Yeboah, P. Puplampu, J. Ainuson, J. Akpalu, B. Gyan, and A. G. B. Amoah, "Peripheral artery disease and exertional leg symptoms in diabetes patients in Ghana," BMC Cardiovascular Disorders, vol. 16, no. 1, p. 68, 2016.

[2] K. Yeboah, P. Puplampu, V. Boima, D. A. Antwi, B. Gyan, and A. G. Amoah, "Peripheral sensory neuropathy in type 2 diabetes patients: a case control study in Accra, Ghana," Journal of Clinical \& Translational Endocrinology, vol. 5, pp. 26-31, 2016.

[3] A. I. Vinik and D. Ziegler, "Diabetic cardiovascular autonomic neuropathy," Circulation, vol. 115, no. 3, pp. 387-397, 2007.

[4] L. Kärvestedt, E. Mårtensson, V. Grill et al., "Peripheral sensory neuropathy associates with micro- or macroangiopathy: results from a population-based study of type 2 diabetic patients in Sweden," Diabetes Care, vol. 32, no. 2, pp. 317322, 2009.

[5] M. J. Fowler, "Microvascular and macrovascular complications of diabetes," Clinical Diabetes, vol. 29, no. 3, pp. 116$122,2011$.

[6] A. I. Vinik, M. T. Holland, J. M. L. Beau, F. J. Liuzzi, K. B. Stansberry, and L. B. Colen, "Diabetic neuropathies," Diabetes Care, vol. 15, no. 12, pp. 1926-1975, 1992.

[7] K. Yeboah, D. A. Antwi, and B. Gyan, "Arterial stiffness in nonhypertensive type 2 diabetes patients in Ghana," International Journal of Endocrinology, vol. 2016, pp. 1-8, 2016.

[8] K. Yeboah, D. A. Antwi, B. Gyan et al., "Arterial stiffness in hypertensive and type 2 diabetes patients in Ghana: comparison of the cardio-ankle vascular index and central aortic techniques," BMC Endocrine Disorders, vol. 16, no. 1, p. 53, 2016.

[9] J. K. Cruickshank, M. Rezailashkajani, and G. Goudot, "Arterial stiffness, fatness, and physical fitness: ready for intervention in childhood and across the life course?" Hypertension, vol. 53, no. 4, pp. 602-604, 2009.

[10] K. Cruickshank, L. Riste, S. G. Anderson, J. S. Wright, G. Dunn, and R. G. Gosling, "Aortic pulse-wave velocity and its relationship to mortality in diabetes and glucose intolerance: an integrated index of vascular function?" Circulation, vol. 106, no. 16, pp. 2085-2090, 2002.

[11] World Health Organization, Waist Circumference and WaistHip Ratio: Report of a WHO Expert Consultation, WHO Document Production Services, Geneva, Switzerland, 2011.

[12] W. T. Friedewald, R. I. Levy, and D. S. Fredrickson, "Estimation of the concentration of low-density lipoprotein cholesterol in plasma, without use of the preparative ultracentrifuge," Clinical Chemistry, vol. 18, no. 6, pp. 499-502, 1972.

[13] I. G. Horvath, A. Nemeth, Z. Lenkey et al., "Invasive validation of a new oscillometric device (Arteriograph) for measuring augmentation index, central blood pressure and aortic pulse wave velocity," Journal of Hypertension, vol. 28, no. 10, pp. 2068-2075, 2010.

[14] J. Sugawara, K. Hayashi, T. Yokoi, and H. Tanaka, "Ageassociated elongation of the ascending aorta in adults," JACC Cardiovascular Imaging, vol. 1, no. 6, pp. 739-748, 2008.

[15] J. Sugawara, K. Hayashi, T. Yokoi, and H. Tanaka, "Carotidfemoral pulse wave velocity: impact of different arterial path length measurements," Artery Research, vol. 4, no. 1, pp. 27$31,2010$.

[16] C.-K. Sun, "Cardio-ankle vascular index (CAVI) as an indicator of arterial stiffness," Integrated Blood Pressure Control, vol. 6, p. 27, 2013.

[17] K. Shirai, J. Utino, A. Saiki et al., "Evaluation of blood pressure control using a new arterial stiffness parameter, cardio-ankle vascular index (CAVI)," Current Hypertension Reviews, vol. 9, no. 1, pp. 66-75, 2013.

[18] H. Yokoyama, Y. Yokota, J. Tada, and S. Kanno, "Diabetic neuropathy is closely associated with arterial stiffening and thickness in type 2 diabetes," Diabetic Medicine, vol. 24, no. 12, pp. 1329-1335, 2007.

[19] E. S. Kim, S.-d. Moon, H.-S. Kim et al., "Diabetic peripheral neuropathy is associated with increased arterial stiffness without changes in carotid intima-media thickness in type 2 diabetes," Diabetes Care, vol. 34, no. 6, pp. 1403-1405, 2011. 
[20] A. Tentolouris, I. Eleftheriadou, P. Grigoropoulou et al., "The association between pulse wave velocity and peripheral neuropathy in patients with type 2 diabetes mellitus," Journal of Diabetes and its Complications, vol. 31, no. 11, pp. 1624-1629, 2017.

[21] C. R. L. Cardoso, C. B. M. Moran, F. S. Marinho, M. T. Ferreira, and G. F. Salles, "Increased aortic stiffness predicts future development and progression of peripheral neuropathy in patients with type 2 diabetes: the Rio de Janeiro Type 2 Diabetes Cohort Study," Diabetologia, vol. 58, no. 9, pp. 2161-2168, 2015.

[22] D. R. Smith, A. I. Kobrine, and H. V. Rizzoli, "Absence of autoregulation in peripheral nerve blood flow," Journal of the Neurological Sciences, vol. 33, no. 3, pp. 347-352, 1977.

[23] M. E. Edmonds, N. Morrison, J. W. Laws, and P. J. Watkins, "Medial arterial calcification and diabetic neuropathy," British Medical Journal, vol. 284, no. 6320, pp. 928-930, 1982.

[24] M. J. Young, J. E. Adams, G. F. Anderson, A. J. M. Boulton, and P. R. Cavanagh, "Medial arterial calcification in the feet of diabetic patients and matched non-diabetic control subjects," Diabetologia, vol. 36, no. 7, pp. 615-621, 1993.

[25] W. J. Jeffcoate, L. M. Rasmussen, L. C. Hofbauer, and F. L. Game, "Medial arterial calcification in diabetes and its relationship to neuropathy," Diabetologia, vol. 52, no. 12, pp. 2478-2488, 2009.

[26] M. Young, C. Manes, and A. Boulton, "Vibration perception threshold predicts foot ulceration: a prospective study," Diabetic Med, vol. 9, Supplement 2, p. 542, 1992.

[27] L. Karvestedt, E. Martensson, V. Grill et al., "Peripheral sensory neuropathy associates with micro- or macroangiopathy: results from a population-based study of type 2 diabetic patients in Sweden," Diabetes Care, vol. 32, no. 2, pp. 317-322, 2009.

[28] J. Ding, C. Y. Cheung, M. K. Ikram et al., "Early retinal arteriolar changes and peripheral neuropathy in diabetes," Diabetes Care, vol. 35, no. 5, pp. 1098-1104, 2012.

[29] A. J. Boulton, A. I. Vinik, J. C. Arezzo et al., "Diabetic neuropathies: a statement by the American Diabetes Association," Diabetes Care, vol. 28, no. 4, pp. 956-962, 2005.

[30] V. Bril and B. A. Perkins, "Comparison of vibration perception thresholds obtained with the neurothesiometer and the CASE IV and relationship to nerve conduction studies," Diabetic Medicine, vol. 19, no. 8, pp. 661-666, 2002.

[31] T. Bergenheim, B. Borssén, and F. Lithner, "Sensory thresholds for vibration, perception and pain in diabetic patients aged 15-50 years," Diabetes Research and Clinical Practice, vol. 16, no. 1, pp. 47-52, 1992.

[32] S. Tesfaye, N. Chaturvedi, S. E. M. Eaton et al., "Vascular risk factors and diabetic neuropathy," New England Journal of Medicine, vol. 352, no. 4, pp. 341-350, 2005.

[33] S. Yagihashi, H. Yasuda, H. Sasaki et al., "Prevalence and clinical staging of diabetic polyneuropathy and its risk factors in Japanese diabetic patients. Validation by electrophysiology and skin biopsy," in Journal of the Peripheral Nervous System: 2011, pp. S152-S153, Wiley-Blackwell, Commerce place, 350 main St, Malden 02148, MA USA, 2011.
[34] A. P. Garrow and A. J. M. Boulton, "Vibration perception threshold-a valuable assessment of neural dysfunction in people with diabetes," Diabetes/Metabolism Research and Reviews, vol. 22, no. 5, pp. 411-419, 2006.

[35] S. Tesfaye, A. J. M. Boulton, P. J. Dyck et al., "Diabetic neuropathies: update on definitions, diagnostic criteria, estimation of severity, and treatments," Diabetes Care, vol. 33, no. 10, pp. 2285-2293, 2010. 


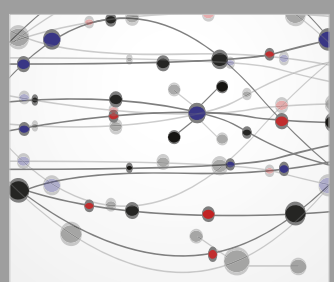

The Scientific World Journal
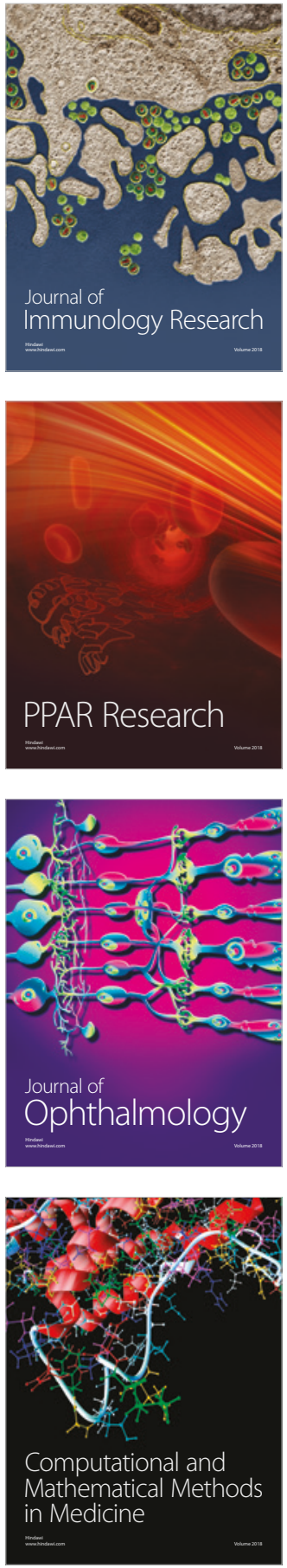

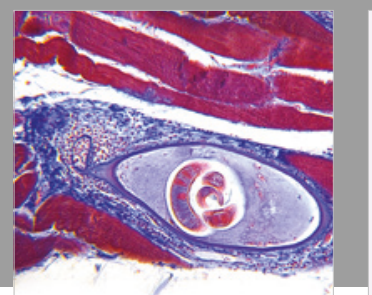

Gastroenterology Research and Practice

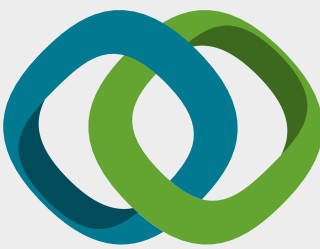

\section{Hindawi}

Submit your manuscripts at

www.hindawi.com
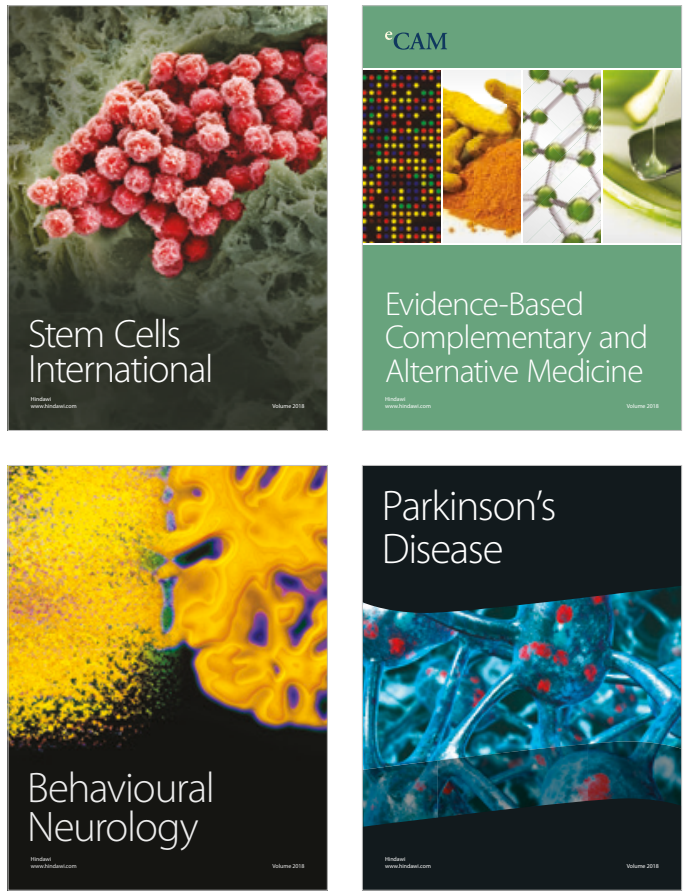

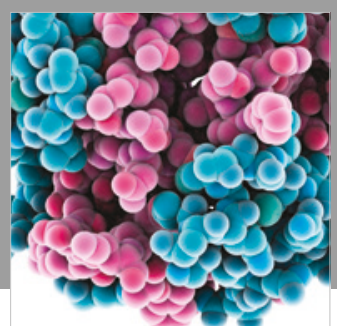

ournal of

Diabetes Research

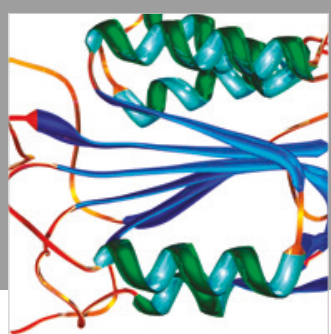

Disease Markers
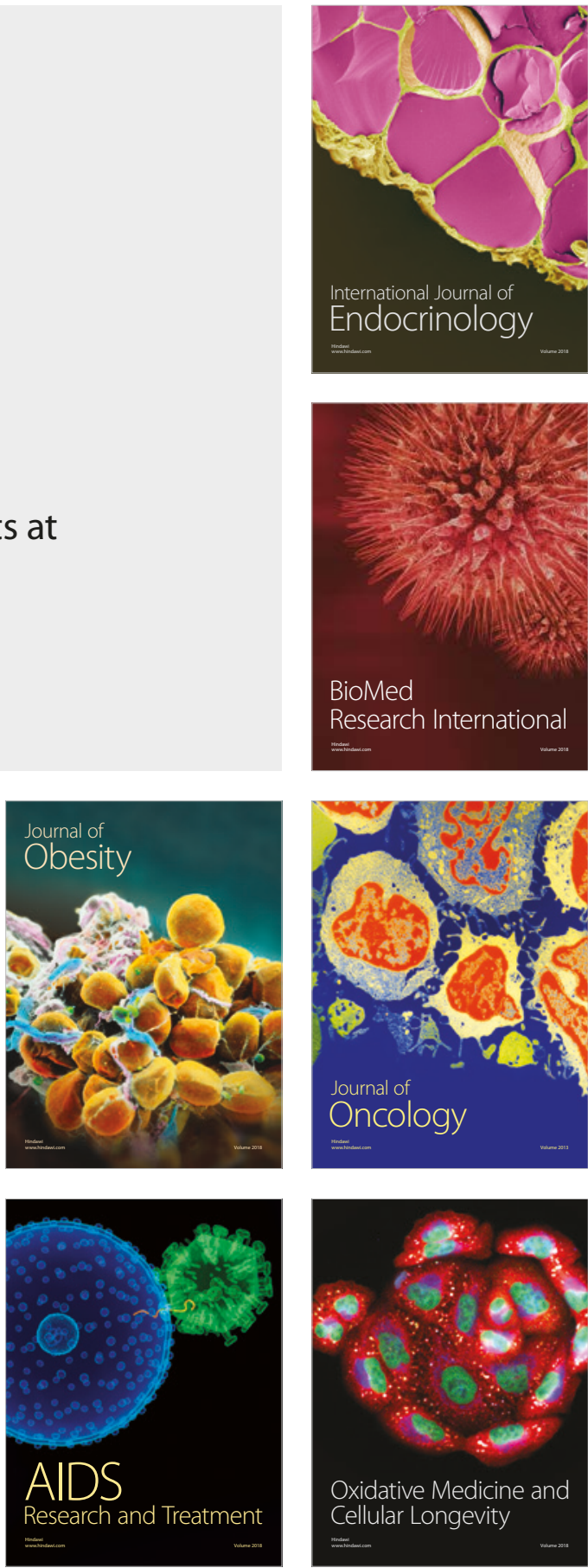